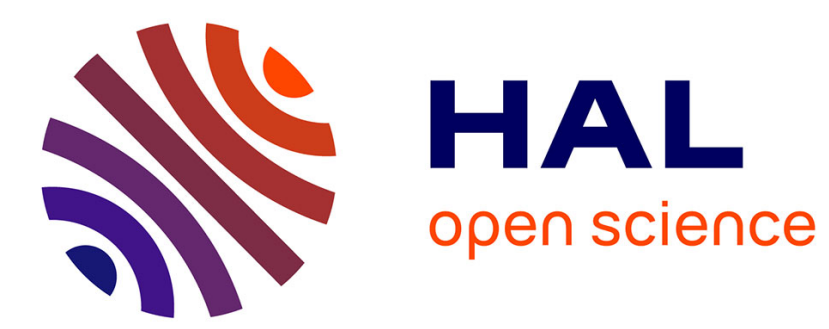

\title{
Hermann, Rau, Mangoldt : les origines de la fonction d'offre de marché en Allemagne (1830 - 1870)
}

\author{
Paola Tubaro
}

\section{To cite this version:}

Paola Tubaro. Hermann, Rau, Mangoldt : les origines de la fonction d'offre de marché en Allemagne

(1830 - 1870). Recherches Economiques de Louvain - Louvain economic review, 2005, 71 (2), pp.223

- 243. 10.3917/rel.712.0223. hal-01648314

\section{HAL Id: hal-01648314 \\ https://hal.science/hal-01648314}

Submitted on 28 Nov 2020

HAL is a multi-disciplinary open access archive for the deposit and dissemination of scientific research documents, whether they are published or not. The documents may come from teaching and research institutions in France or abroad, or from public or private research centers.
L'archive ouverte pluridisciplinaire HAL, est destinée au dépôt et à la diffusion de documents scientifiques de niveau recherche, publiés ou non, émanant des établissements d'enseignement et de recherche français ou étrangers, des laboratoires publics ou privés. 


\title{
HERMANN, RAU, MANGOLDT : LES ORIGINES DE LA FONCTION D'OFFRE DE MARCHÉ EN ALLEMAGNE (1830 - 1870)
}

Paola Tubaro

\author{
De Boeck Supérieur | «Recherches économiques de Louvain »
}

2005/2 Vol. 71 | pages 223 à 243

ISSN 0770-4518

ISBN 2-8041-4747-9

Article disponible en ligne à l'adresse :

https://www.cairn.info/revue-recherches-economiques-delouvain-2005-2-page-223.htm

\section{Pour citer cet article :}

Paola Tubaro, « Hermann, Rau, Mangoldt : les origines de la fonction d'offre de marché en Allemagne (1830 - 1870) », Recherches économiques de Louvain 2005/2 (Vol. 71), p. 223-243.

DOI 10.3917/rel.712.0223

Distribution électronique Cairn.info pour De Boeck Supérieur.

(C) De Boeck Supérieur. Tous droits réservés pour tous pays.

La reproduction ou représentation de cet article, notamment par photocopie, n'est autorisée que dans les limites des conditions générales d'utilisation du site ou, le cas échéant, des conditions générales de la licence souscrite par votre établissement. Toute autre reproduction ou représentation, en tout ou partie, sous quelque forme et de quelque manière que ce soit, est interdite sauf accord préalable et écrit de l'éditeur, en dehors des cas prévus par la législation en vigueur en France. Il est précisé que son stockage dans une base de données est également interdit. 


\title{
Hermann, Rau, Mangoldt : les origines de la fonction d'offre de marché en Allemagne (1830 - 1870)
}

\author{
Paola Tubaro* \\ P.H.A.R.E., Université Paris X-Nanterre**
}

\section{Introduction}

Alfred Marshall employa systématiquement l'analyse en termes d'offre et de demande, pour mettre en évidence les diverses configurations qui peuvent être envisagées et pour écarter l'idée d'une cause unique (le coût de production ou l'utilité) capable d'expliquer la valeur des marchandises. En particulier, il utilisait une fonction d'offre représentant, pour une marchandise donnée, la relation entre le prix et la quantité mise en vente. Elle s'appuyait sur l'analyse des coûts de production : elle était croissante, invariante ou décroissante selon que le coût de production augmentait, restait constant ou diminuait avec la quantité produite.

Piero Sraffa (1925) souligna la divergence entre cette approche et celle des auteurs classiques, pour qui la relation entre prix et quantités n'existait qu'en déséquilibre. Leur analyse des coûts décroissants (liée à l'étude de la division du travail) relevait du domaine de la production, tandis que celle des coûts croissants faisait partie de celui de la répartition (théorie de la rente), ces deux tendances n'étant pas fusionnées en une loi unique des coûts de production non proportionnels, pouvant être vue comme le fondement d'une théorie des prix d'équilibre. Sraffa expliqua l'importance

* Une première version de cet article a été présentée au colloque de l'Association Charles Gide pour l'étude de la pensée économique, qui a eu lieu à Grenoble en septembre 2003. Je dois beaucoup à ceux qui, par leurs réflexions critiques et leurs conseils, m'ont permis de l'améliorer. Je tiens en particulier à remercier Carlo Benetti, Bertram Schefold, Alain Béraud et Pascal Bridel, ainsi qu'un rapporteur des Recherches économiques de Louvain. Je reste bien sûr seule responsable des insuffisances qui subsistent.

** P.H.A.R.E. - Université Paris X - Nanterre - Bâtiment K, Bureau K 131, 200, avenue de la République, 92001 Nanterre cedex, France - Tél. : +3314097 7543 - Fax :+3314097 7057 - E-mail : paola.tubaro@uparis10.fr 
accordée par les économistes d'inspiration marshallienne à l'idée d'un prix toujours dépendant de la quantité produite sur la base de l'influence de la théorie de l'utilité, à partir des années 1870 : la fonction d'offre néoclassique serait une conséquence du glissement de la théorie de la valeur, ayant initialement son centre dans la théorie de la production, vers l'utilité. La recherche sur l'utilité marginale ayant attiré l'attention sur la relation entre prix et quantité consommée, valable à l'équilibre aussi bien qu'en déséquilibre, les économistes auraient été incités à formuler l'idée symétrique d'une relation entre prix et quantité produite.

Mais cette explication ne tient pas compte des travaux des précurseurs : il existe des contributions qui précèdent la mise au point de la théorie néoclassique sous sa forme achevée, qui montrent que l'idée qu'il existe, à l'équilibre et en déséquilibre, une relation entre prix et quantité produite, avait été formulée et exprimée mathématiquement bien avant les développements de la théorie de l'utilité marginale auxquels pensait Sraffa. Trois ouvrages allemands (connus par Marshall, qui séjourna à Dresde en 1868 et à Berlin en 1870-1) nous semblent particulièrement significatifs : les Staatswirthschaftliche Untersuchungen (1832) de Friedrich von Hermann, les Grundsätze der Volkswirthschaftslehre (1841) de Karl Heinrich Rau, et le Grundriß der Volkswirthschaftslehre (1863) de Hans von Mangoldt.

Nous allons présenter ces contributions, en essayant d'en illustrer l'originalité et l'intérêt, afin de mieux comprendre les origines de l'approche néoclassique, surtout de sa variante marshallienne. Nous chercherons les raisons pouvant avoir poussé ces auteurs à développer l'idée d'une relation entre prix et quantité produite, ainsi que sur les facteurs préfigurant le passage de la vision classique des phénomènes économiques à la néoclassique. Nous étudierons également les avantages et les limites de leur utilisation des mathématiques.

Nous verrons que la relation entre prix et quantité produite, avant la « révolution marginaliste », tire ses origines d'une réinterprétation des thèses classiques. En particulier, les recherches de Hermann, Rau et Mangoldt sur le cas d'une relation croissante entre prix et quantité produite sont calquées sur l'étude des coûts de production croissants, faite par David Ricardo pour analyser le phénomène de la rente. Cela a conduit graduellement à un changement de perspective par rapport à la vision classique, même en l'absence de développements poussés de la théorie de l'utilité.

L'outillage mathématique employé par ces trois auteurs inclut l'algèbre (équations et inégalités) de Hermann et la géométrie analytique (courbes dans un repère prix - quantité) de Rau et Mangoldt. Quoique ces premières formalisations et représentations graphiques puissent apparaître fort simples et incomplètes aux lecteurs d'aujourd'hui, elles sont quand même intéressantes, parce que ces instruments ont été utilisés ensuite par l'école marshallienne, et demeurent aujourd'hui d'usage courant pour l'économiste. D'autre part, nous verrons que ces tentatives de mathématisation ont contribué à l'évolution de la théorie économique, en favorisant la transition graduelle 
de la pensée classique à l'affirmation de l'école néoclassique. Alors que la théorie classique, dont les trois auteurs considérés s'inspiraient, fait ressortir l'hétérogénéité des phénomènes sous-jacents à la forme des fonctions de coût, les instruments mathématiques qu'ils utilisèrent en suggèrent plutôt une interprétation en termes de symétrie et d'unité : la formalisation algébrique de Hermann s'adapte à décrire de diverses situations, et les graphiques de Rau et Mangoldt représentent les différents cas (coûts constants, croissants, décroissants, non pertinents) comme des courbes ayant des caractéristiques semblables car, géométriquement, la pente de la courbe d'offre est la seule propriété qui change de l'un à l'autre; les frontières entre eux deviennent assez floues. Ces contributions à l'économie mathématique préfigurent ainsi l'idée marshallienne d'une loi unique, pouvant expliquer toute forme de la courbe d'offre.

Notre premier paragraphe traite la formalisation algébrique de Hermann. La deuxième et la troisième partie sont consacrées aux diagrammes de Rau et Mangoldt respectivement. Les conclusions sont présentées dans la dernière section. La traduction française des passages cités est la nôtre.

\section{La formalisation algébrique de Hermann}

Friedrich Benedikt Wilhelm von Hermann (1795 - 1868) mathématicien de formation, fut professeur de mathématiques et ensuite d'économie à $\mathrm{Mu}$ nich, ainsi que conseiller du roi de Bavière. Dans ses Staatswirthschaftliche Untersuchungen, il essaya d'approfondir l'analyse de questions, comme la théorie du capital et de la répartition, traités à son avis de façon insatisfaisante par les économistes précédents. Il n'employa aucun graphique dans son ouvrage, mais se servit de l'algèbre pour discuter la formation du prix de production d'une marchandise; l'idée d'une courbe d'offre croissante qui en est issue fut reprise dans plusieurs manuels allemands de l'époque, comme le souligne Erich Streissler (1997).

\subsection{L'équation du coût de production}

L'offre des marchandises dépend, selon Hermann, en plus des facteurs naturels ou sociaux qui favorisent ou empêchent la concurrence, des coûts de production. Ces derniers se composent de deux éléments: d'une part, du capital circulant, c'est-à-dire de l'ensemble des biens, avancés au début de la période de production, dont la valeur se transfère entièrement au produit final. Il s'agit des biens - salaire (la paie de l'entrepreneur pour son travail de direction ainsi que les salaires des travailleurs embauchés par l'entreprise), des matières premières et des produits intermédiaires, aussi bien que de la somme correspondante à la valeur de l'usure annuelle du capital fixe (bâtiments, machines, etc.), qui doit être mise de côté chaque année pour 
remplacer ce capital fixe à la fin de sa dernière année d'utilisation (Hermann (1832), p. 79). D'autre part, il y a les « services du capital », à savoir le profit rapporté par la totalité du capital avancé, fixe et circulant, qui représente une sorte de coût d'opportunité pour le producteur, et exprime la contribution du capital total à l'obtention du produit : « Comme la valeur d'usage du bien dépend du renoncement du producteur à utiliser les services du capital pour sa propre consommation, il faut que la valeur d'échange du bien inclue tous les services du capital auxquels on a renoncé pour la production » (Hermann (1832), p. 79).

Par conséquent, « On peut dire alors brièvement que le coût de chaque produit est égal à la somme des capitaux qui entrent directement dans le produit plus la valeur du service de tous les capitaux employés dans la production » (Hermann (1832), pp. 79 - 80). En langage formel : « Soit $A$ le capital circulant, qui se transmet au produit, et $B$ le capital fixe employé dans la production; soit $\frac{p}{100}$ la valeur moyenne des services du capital, le coûts est alors :

$$
A+(A+B) \frac{p}{100} \quad \gg(\text { Hermann (1832), p. 80). }
$$

Afin de mieux comprendre la formule de Hermann, essayons de la traduire en langage moderne, tout en conservant le sens que lui a voulu donner l'auteur. Considérons le cas d'une marchandise $i$ quelconque, dont une unité est produite à l'aide d'une quantité $A_{i}$ de capital circulant et d'une quantité $B_{i}$ de capital fixe. Appelons $r$ le taux de profit qui est selon Hermann, comme chez les classiques anglais, uniforme pour l'économie dans son ensemble, en raison de l'hypothèse d'absence de barrière à l'entrée dans toutes les branches (Hermann (1832), p. 82). Le coût de production $p_{i}$ de la marchandise $i$ est alors égal à :

$$
p_{i}=A_{i}+\left(A_{i}+B_{i}\right) r
$$

Le premier terme $\left(A_{i}\right)$ correspond à la dépense en capital circulant, et l'autre $\left(\left(A_{i}+B_{i}\right) r\right)$ représente le profit obtenu par le capitaliste sur la totalité du capital avancé, au taux normal de profit. L'équation (1) peut être réécrite sous la forme:

$$
p_{i}=A_{i}(1+r)+B_{i} r
$$

Il y a là une ambiguïté car, comme nous l'avons vu, Hermann écrit expressément que le capital circulant inclut la dépréciation. En symboles (en supposant pour simplifier que la dépense pour les matières premières et les produits intermédiaires est nulle) :

$$
A_{i}=w L_{i}+D_{i}
$$


Les symboles $w$ et $L_{i}$ indiquent le taux de salaire et la quantité de travail nécessaire pour produire une unité de la marchandise $i$ respectivement, et la variable $D_{i}$ désigne la dépréciation du capital fixe utilisé pour la production de $i$. L'équation (1) de Hermann est alors inexacte, comme le remarque Heinz Kurz ((1998a), p. 97). La formule correcte est:

$$
p_{i}=w L_{i}(1+r)+D_{i}+B_{i} r
$$

Alternativement, en tenant compte de (3) :

$$
p_{i}=A_{i}(1+r)+\left(B_{i}-D_{i}\right) r
$$

La formule de Hermann devrait donc être remplacée par notre équation (4) ou (5), à moins que le capital fixe ne soit éternel.

L'équation (4) pourrait aussi être écrite sous la forme:

$$
p_{i}+\left(B_{i}-D_{i}\right)=w L_{i}(1+r)+B_{i}(1+r)
$$

Autrement dit, le capital fixe utilisé peut être vu comme un produit joint du processus de production. Hermann partageait l'idée de Robert Torrens que « Partout, les mêmes capitaux au même moment donnent les mêmes produits (en prenant pour produit la valeur conjointe du résultat total de la production et des résidus des capitaux)» (Hermann (1832), p. 40).

\subsection{La comparaison de deux techniques différentes}

Supposons qu'une nouvelle méthode de production de la marchandise $i$ devient disponible. Entre les deux techniques, le producteur choisit celle qui minimise son coût de production : « Soit le coût $A+(A+B) \frac{p}{100}$, comme auparavant; soit $a$ la réduction du capital circulant, due au fait qu'un nouveau capital fixe $B^{\prime}$ (par exemple une machine) est mis en œuvre, la dépréciation annuelle de ce dernier, qui s'ajoute au capital circulant, étant égale à $b$; le nouveau coût est alors $A-a+b+(A-a+b+B+B) \frac{p}{100}$, et il doit être inférieur au coût précédent, à savoir $b+(B+b) \frac{p}{100}<a+a \frac{p}{100}$. Le changement est avantageux lorsque $p<\frac{100(a-b)}{B+b-a}$, ou $b<\frac{a(100+p)-B p}{100+p}$, ou encore $a>\frac{(100+p)+B p}{100+p}$, ou $B<\frac{(a-b)(100+p)}{p} »$ (Hermann (1832), pp. 87-88).

Essayons d'exprimer ces concepts en termes modernes. La nouvelle technique $\left(A_{i}^{1}, B_{i}^{1}\right)$ se distingue de celle qui la précède $\left(A_{i}, B_{i}\right)$ par l'utilisation d'une quantité supérieure de capital fixe : $B_{i}^{1}=B_{i}+B^{\prime}$, à savoir, le nouveau capital fixe se compose du capital fixe précédent plus une nouvelle machine, ce qui implique $B_{i}^{1}>B_{i}$. L'introduction d'une machine conduit généralement à une réduction de la quantité de capital circulant, par exemple en raison du fait qu'elle remplace une partie des travailleurs:si l'on appelle $a_{i}$ cette réduction du capital circulant, cela veut dire $A_{i}-a_{i}<A_{i}$. 
C'est le même problème qu'analysa Ricardo dans le chapitre 31 des Principles mais, chez Hermann, l'hypothèse que l'introduction d'un nouveau capital fixe remplace une partie du capital circulant n'implique pas qu'une mécanisation de plus en plus poussée de la production provoque l'apparition du chômage dans l'économie, du moins à long terme, car des mécanismes de compensation se mettent en place. La mise en œuvre d'une machine équivaut à une augmentation du potentiel productif de l'économie qui, conformément à la loi de Say, entraîne une hausse de la demande de biens et, par conséquent, un accroissement de la demande de travail. Ainsi, les travailleurs qui avaient initialement perdu leur emploi sont recrutés à nouveau, parfois dans des branches différentes de celle d'origine, de telle sorte que l'effet final est positif pour tous (Hermann (1832), p. 282).

Il est intéressant de remarquer que cette question est algébriquement indécidable, étant donné que l'on ne peut pas déduire de l'inégalité $A_{i}-a_{i}<A_{i}$ que le capital circulant total diminue suite à la mise en ouvre de la machine, de telle sorte que $A_{i}^{1}<A_{i}$, car la dépréciation $b_{i}$ s'ajoute au capital circulant annuellement nécessaire pour produire une unité de la marchandise $i$ avec la nouvelle technique : puisque $A_{i}^{1}=A_{i}-a_{i}+b_{i}$, le signe de la différence $A_{i}^{1}-A_{i}$ est a priori indéterminé. Le coût de production relatif à la nouvelle technique est:

$$
p_{i}^{1}=A_{i}^{1}(1+r)+\left(B_{i}^{1}-b_{i}\right) r
$$

En remplaçant $A_{i}^{1}$ et $B_{i}^{1}$ par leurs valeurs, et en supposant que la partie du capital fixe qui est utilisée dans les deux techniques, $B_{i}$, est éternelle $\left(D_{i}=0\right)$, on obtient :

$$
p_{i}^{1}=\left(A_{i}-a_{i}\right)(1+r)+b_{i}+\left(B_{i}+B\right) r
$$

L'équation (8) diffère de celle qui est donnée par Hermann lui-même, qui est (exprimée dans les symboles plus modernes utilisés ici) :

$$
p_{i}^{1}=\left(A_{i}-a_{i}+b_{i}\right)(1+r)+\left(B_{i}+B\right) r
$$

Il y a donc une deuxième imprécision dans le calcul de la dépréciation du capital fixe. Les équations (8) et (9) ne sont égales que lorsque la nouvelle machine est éternelle $\left(b_{i}=0\right)$.

Voyons maintenant le problème du choix technique. Le producteur passe de la technique initiale $\left(A_{i}, B_{i}\right)$ à une autre $\left(A_{i}^{1}, B_{i}^{1}\right)$, si son coût de production diminue, c'est-à-dire si $p_{i}^{1}<p_{i}$. Autrement dit, sous l'hypothèse $D_{i}=0$, la nouvelle technique est avantageuse si :

$$
p_{i}^{1}=\left(A_{i}-a_{i}\right)(1+r)+b_{i}+\left(B_{i}+B\right) r<A_{i}(1+r)+B_{i} r=p_{i}
$$

Avec des simples transformations algébriques, on obtient:

$$
b_{i}+B^{\prime} r<a_{i}(1+r)
$$


Le passage à la nouvelle technique $\left(A_{i}^{1}, B_{i}^{1}\right)$ est rentable si l'accroissement du coût de production, dû à l'introduction de la machine $\left(b_{i}+B^{\prime} r\right)$ est en valeur absolue inférieur à la réduction du coût de production engendrée par la substitution du capital fixe à une partie du capital circulant $\left(a_{i}(1+r)\right)$.

Hermann partageait l'idée de Ricardo que le choix technique dépend du taux de profit: « cette possibilité de réduction des coûts dépend du taux de profit, et joue d'autant plus que le profit normal est bas. C'est pourquoi on la trouve surtout dans les pays où le taux de profit est bas par rapport au salaire. Les machines de l'Angleterre et de l'Amérique du Nord, qui remplacent le travail manuel, qui est très cher là-bas, en sont des exemples » (Hermann (1832), p. 88). Hermann ne semble pas avoir considéré la possibilité que le taux général de profit se modifie suite à un changement de technique : il considérait la variable $r$ (ou, pour utiliser ses propres symboles, $\left.\frac{p}{100}\right)$ comme une donnée, et se bornait à considérer les effets d'un choix technique sur le prix de la marchandise $i$ en question, sans se demander si cela peut avoir des conséquences sur l'économie dans son ensemble.

\subsection{Les applications possibles de la formalisation de Hermann : les coûts constants}

Nous allons nous servir de la formalisation de Hermann pour élucider les différents cas possibles, en commençant par les coûts constants. Reprenons la comparaison entre deux techniques : si la condition $p_{i}^{1}<p_{i}$ est vérifiée, tout producteur de la marchandise $i$ souhaite adopter la technique $\left(A_{i}^{1}, B_{i}^{1}\right)$. Il faut se demander alors si cette technique peut toujours être appliquée sans limites par tous, quels que soient les niveaux d'activité. La réponse à cette question peut varier d'une branche à l'autre, d'une technique à l'autre, et d'un moment dans le temps à un autre : elle dépend de la disponibilité des ressources de type $A$ et $B$ qui entrent dans la production de la marchandise $i$.

Lorsque la quantité de ces ressources peut être augmentée sans limites, du moins à long terme, la technique la moins chère $\left(A_{i}^{1}, B_{i}^{1}\right)$ va être utilisée par tous, et le prix de la marchandise $i$ s'établit au niveau $p_{i}^{1}$. Dans ce cas, le prix d'équilibre est déterminé par l'offre, et la quantité par la demande, conformément à la vision classique de la formation des prix des produits manufacturés, dans une perspective de long terme.

Hermann exprime ces idées sans formules, en privilégiant la forme littéraire: «Si la cause de la baisse du prix est une réduction du coût de production d'autrui, ou le fait que notre marchandise est en concurrence avec une autre, moins chère, si n'importe quelle quantité de cette dernière peut être apportée au marché, le prix ne va pas monter, car la production et l'offre de la marchandise la plus chère doivent diminuer; elle est supplantée par la nouvelle marchandise, moins chère, et l'ancienne méthode de production doit changer, c'est-à-dire s'interrompre, ou être remplacée par une méthode plus rentable» (Hermann (1832), p. 82). 


\subsection{Les coûts croissants}

Reprenons la formalisation du choix technique de Hermann, afin d'en montrer d'autres applications possibles. Lorsque l'une des ressources est disponible en quantité limitée (Hermann (1832), p. 85), la technique $\left(A_{i}^{1}, B_{i}^{1}\right)$ ne peut pas être généralisée; il y a alors deux possibilités. Si le produit total obtenu avec la technique la moins chère $\left(A_{i}^{1}, B_{i}^{1}\right)$ est suffisant pour satisfaire la demande globale, seulement cette technique va être utilisée, et le prix de la marchandise $i$ s'établit au niveau $p_{i}^{1}$. Mais si le produit ainsi obtenu est insuffisant, une partie de la demande globale doit être satisfaite à l'aide de l'autre technique $\left(A_{i}, B_{i}\right)$. Le prix s'établit alors au niveau $p_{i}>p_{i}^{1}$, juste suffisant pour couvrir le coût de production des unités les plus chères. Cette explication est donnée en langage littéraire par Hermann : «Si l'on ne peut pas satisfaire la demande totale avec la marchandise produite aux coûts les plus bas, au début le nouveau produit va provoquer une baisse du prix, et attirer une partie des acheteurs de la marchandise la plus chère. Celle-ci est exclue du marché, et une partie de la demande reste insatisfaite, ce qui va faire monter le prix jusqu'à ce que la quantité offerte de la marchandise la plus chère couvre entièrement la demande » (Hermann (1832), p. 83).

Au-delà d'un certain niveau, une hausse de la production (qui par exemple se rend souhaitable en raison d'une augmentation exogène de la demande) n'est possible qu'à des coûts croissants. C'est, selon Ricardo, le cas du blé : la quantité disponible de terre cultivable ne pouvant pas être augmentée, pour augmenter la production de blé il faut soit avoir recours à des terres moins fertiles ou moins bien situées, soit augmenter les doses de capital et de travail appliquées sur la même terre, ce qui donne lieu à une hausse du produit moins que proportionnelle par rapport à l'augmentation de la dépense pour l'achat des inputs.

Comme le remarquent Streissler (1997, 1999) et Kurz (1998a, 1998b), une telle possibilité ne concerne pas que l'agriculture chez Hermann, mais aussi les manufactures, l'analyse ricardienne de la terre pouvant être généralisée à n'importe quel type de capital fixe. La rareté n'est jamais absolue, mais relative; il n'est pas impossible d'augmenter la quantité de terre à cultiver, comme le cas hollandais le montre, mais cela peut être extrêmement dispendieux. Tous les facteurs sont augmentables, à des coûts plus ou moins élevés. Les capitaux fixes ressemblent à la terre : ils doivent être considérés comme des biens rares, dont la quantité ne peut pas facilement être modifiée, du moins à court terme. La loi de l'uniformité du taux de profit s'applique au capital circulant, qui généralement peut être transféré assez rapidement d'un secteur à l'autre. Il n'en va pas de même pour le capital fixe : «L'hypothèse de l'égalisation des taux de profit repose sur l'idée que le capital peut être librement et facilement transféré d'une activité à l'autre. Il est clair que cela ne se vérifie que pour le capital circulant, car celuici revient après la vente du produit sous la forme indifférente de l'argent, qui permet toute sorte d'utilisation. Seulement dans ce cas le rapport de la valeur des services à celle de la masse du capital est partout le même » (Her- 
mann (1832), p. 149). Le gain qu'un capital fixe rapporte à son propriétaire n'est pas un profit à proprement parler, mais ressemble plutôt à une rente. Comme le souligne Kurz, Hermann anticipa ainsi la notion marshallienne de quasi-rente ((1998a), p. 101).

La rareté d'un bien ne dépend pas de facteurs naturels, mais d'éléments techniques et économiques; ainsi est-elle introduite dans presque toutes les industries, ce qui conduit à la conclusion que : «De cette recherche sur les coûts résulte que la proposition selon laquelle le prix tend toujours à se rapprocher du coût, et ne peut pas rester au-dessus ou en dessous de celui-ci pour longtemps, n'est pas tout à fait vraie, du moins au niveau de généralité qui lui est attribué, en faisant abstraction des autres circonstances qui influent sur la formation du prix. Tout particulièrement le point, au-dessus ou en dessous duquel le prix ne peut pas rester longtemps, est le coût de la partie de la masse totale d'un produit, qui est obtenue à l'aide des moyens de production les moins efficaces, ou sous les conditions les plus défavorables, et qui est nécessaire pour couvrir la totalité de la demande » (Hermann (1832), p. 88). Il s'agit là d'une formulation très claire de la notion de coût marginal.

La démarche de Hermann, qui tire ses origines de la théorie ricardienne de la rente, met en relief la participation de la demande à la détermination du prix de la plupart des marchandises : «Le premier et le plus important élément déterminant le prix est en tous les cas la demande, qui dépend essentiellement de la valeur d'usage du bien et du pouvoir d'achat des acquéreurs. La demande et ce que les acheteurs sont disposés à donner en échange d'un bien déterminent ce qu'ils acceptent de sacrifier pour obtenir la chose désirée, ainsi que le niveau du coût de la production la moins efficace » (Hermann (1832), p. 95). L'asymétrie constatée dans le cas précédent (l'offre déterminant seule le prix, et la demande déterminant seule la quantité) laisse la place ici à la symétrie : offre et demande participent ensemble à l'établissement de la quantité et du prix d'équilibre.

Dans ces conditions, la répartition du revenu dépend en dernière analyse de la demande: «L'entrepreneur achète du travail non pas pour sa propre consommation, mais pour le revendre sous la forme d'un produit; il agit au nom des consommateurs. Ce que ceux-ci donnent pour le produit contient la vraie rémunération de la prestation des travailleurs [...]. L'entrepreneur, avec son capital, n'est que l'intermédiaire de l'échange entre le consommateur et le travailleur [...]. Il [le travail] a toujours pour but de satisfaire immédiatement un besoin, et doit être rémunéré par le revenu du consommateur. Ainsi disparaît la différence entre services personnels et travaux productifs, qui se fixent en une œuvre» (Hermann (1832), p. 281).

Le raisonnement de Hermann se réfère aux conditions de la branche dans son ensemble. La courbe d'offre est croissante parce que les différents producteurs d'une même marchandise supportent des coûts de production inégaux : «Lorsque la méthode la plus rentable et la méthode la plus chère sont à la disposition de personnes différentes, le prix va devoir monter jus- 
qu'au coût des biens qui sont obtenus sous les conditions les plus défavorables, que le niveau de la demande rend nécessaire de produire. Dans ce cas, le prix des marchandises obtenues au coût le plus bas va rester longtemps au-dessus de leur coût de production. Si sur un marché, approvisionné avec du blé provenant de mauvaises terres, on apportait du blé en provenance de terres meilleures, le prix devrait diminuer : on utiliserait les terres de mauvaise qualité pour produire d'autres denrées à la place du blé, ou on les transformerait en pâturages. Mais si le blé bon marché arrivait à satisfaire seulement une partie du besoin de blé, le prix devrait remonter jusqu'au niveau correspondant au coût de production sur les terres de mauvaise qualité; il serait donc supérieur au coût de production du blé produit au moindre coût » (Hermann (1832), p. 83).

Autrement dit, chaque producteur utilise seulement l'une des techniques existantes, l'accès à la technique la plus rentable étant restreint. Le nombre des producteurs de la branche $i$ étant égal à $N_{i}$, les producteurs $1,2, \ldots, H_{i}\left(H_{i}<N_{i}\right)$ utilisent la technique la meilleure $\left(A_{i}^{1}, B_{i}^{1}\right)$, tandis que les autres $H_{i}+1, \ldots, N_{i}$ utilisent la technique la plus chère $\left(A_{i}, B_{i}\right)$. Le prix de $i$ se fixe alors au niveau $p_{i}$, juste suffisant pour couvrir le coût de production de ceux qui utilisent la technique la plus coûteuse. Les producteurs $1,2, \ldots, H_{i}$, qui supportent des coûts moins élevés, obtiennent un gain supplémentaire, égal à $p_{i}-p_{i}^{1}$ pour chaque unité de marchandise.

Cette construction est formellement identique à la théorie ricardienne de la rente extensive, avec des firmes au lieu des terres, des techniques de production plus ou moins efficaces au lieu de terres de fertilité inégale, et des profits différents au lieu de la rente différentielle : la théorie de l'offre de Hermann est une tentative de généraliser la théorie de la rente à toute branche productive dans laquelle la quantité de l'un des facteurs de production ne peut pas être aisément augmentée. Mais, comme l'a souligné Sraffa (1925), ce raisonnement, valable pour l'étude de la mise en culture de terres de qualité hétérogène, n'est pas toujours applicable à des firmes opérant dans une branche quelconque. Ces dernières ne sont pas comme les terres, qui restent inutilisées lors d'une baisse de la demande de blé, car elles peuvent se déplacer d'une branche à l'autre : les entreprises entrent dans la branche produisant la marchandise $i$ lorsque la demande de cette dernière augmente, et la quittent lorsque la demande baisse. Ces mouvements dépendent en partie de leur efficacité dans la production de $i$, et en partie de leur capacité de modifier rapidement leur production. Lors d'une baisse de la demande de $i$, ce ne sont pas toujours les entreprises les moins efficaces qui quittent la branche, mais aussi celles qui arrivent à transférer leurs capitaux plus facilement - celles qui utilisent des capitaux fixes peu spécialisés, ou qui ont la possibilité de rentrer dans une industrie voisine, pour laquelle le coût de reconversion est bas. Supposons que dans une branche $i$ opère une entreprise $A$ qui obtient un profit annuel égal à 20, et une autre entreprise $B$ dont le profit est égal à 10; dans une autre branche $j, A$ obtiendrait 18 et $B$ aurait 5 : pour aucune d'entre elles n'est convenable de se transférer de $i$ à $j$. Mais si la demande de la marchandise $i$ diminue, celle de $j$ restant constante, de 
telle sorte que le profit annuel de $A$ dans la branche $i$ se réduit à 15 et celui de $B$ à 6 , la firme la plus efficace $A$ est incitée à quitter la branche (et elle va effectivement la quitter si les circonstances le permettent), tandis que l'entreprise $B$ est encouragée à y rester. On voit bien là l'une des difficultés qui émergent lors de tentatives de transposer le schéma ricardien de la rente à d'autres champs d'analyse.

\section{La courbe d'offre de Rau}

Karl Heinrich Rau (1792 - 1870), après des études de sciences économiques et politiques, fut professeur à l'université d'Erlangen et puis de Heidelberg, ainsi que consultant pour l'administration publique. Son ouvrage principal fut un manuel pour l'enseignement supérieur, le Lehrbuch der Politischen Ökonomie, en trois tomes, qui eut un vif succès, avec plusieurs éditions allemandes et de nombreuses traductions. Rau n'employa pas d'algèbre ou d'analyse mathématique mais, dans la quatrième édition du premier tome (Grundsätze der Volkswirthschaftslehre), parue en 1841, il ajouta une annexe, avec un graphique représentant des courbes d'offre et de demande dans un repère prix - quantité. Des diagrammes semblables sont contenus dans une lettre adressée à Adolphe Quetelet, que ce dernier présenta à l'académie de Bruxelles en 1841. Selon Bertram Schefold ((1997), p. XVI) et Christos Baloglou ((1995), p. 160-3), Rau aurait construit son graphique sans connaître la contribution d'Antoine-Augustin Cournot, parue trois ans auparavant.

\subsection{La courbe d'offre verticale}

Étudions le graphique de Rau (fig. 1), en essayant de l'interpréter à la lumière des principes de théorie économique exposés dans les Grundsätze. On peut d'abord remarquer que le diagramme de Rau est construit dans un cadre d'analyse partielle, sans pour autant que ce choix méthodologique soit explicitement mentionné par l'auteur. Le schéma présente deux formes possibles pour la courbe de demande, toujours décroissante, mais qui peut être linéaire $(g h)$ ou non $(i f)$, et deux pour la courbe d'offre, qui est soit verticale $(e d)$, soit croissante $(e k)$. Les quantités sont représentées en abscisses, les prix en ordonnées; les segments horizontaux $a c, a^{\prime} l$, etc. représentent les quantité vendues aux prix $A a, A a^{\prime}$, etc.

Considérons d'abord la courbe d'offre représentée par la droite ed, qui implique que le prix d'équilibre est déterminé uniquement par la demande, et la quantité par l'offre. Une première explication de la forme de cette courbe repose sur la rareté de la marchandise : «Le coût de production d'un bien ne compte pas, si ce bien ne peut pas être reproduit, de telle sorte 


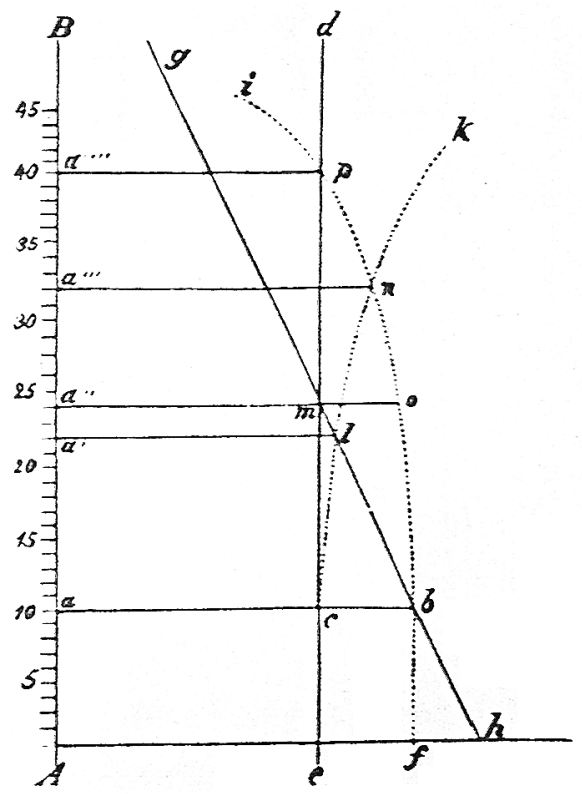

Figure 1 le graphique de Rau ((1841), p. 583).

qu'une unité du bien, offerte sur le marché, ne peut pas facilement être remplacée par une unité équivalente » (Rau (1841), p. 157).

Les paragraphes 169-177 du livre proposent une justification alternative: les différents facteurs qui empêchent, dans de nombreux cas, d'augmenter la production. L'auteur insiste sur l'existence de lois créant des privilèges, des monopoles, des restrictions à la liberté de mouvement; sur la spécialisation des travailleurs et des capitaux (fixes), qui empêche souvent les transferts d'une branche à l'autre; sur les imperfections du marché financier, qui ne permettent pas aux entrepreneurs d'emprunter des montants suffisants pour augmenter la production. Comme le suggère Schefold ((1997), pp. XVIII - XIX) la période temporelle de référence est ici la journée de marché, pendant laquelle la quantité d'une marchandise, pouvant être mise en vente, est fixe, quel que soit son prix. La conception représentée graphiquement par Rau s'avère donc très proche de la notion marshallienne de «période de marché ». Le même graphique (une droite verticale) peut alors correspondre à de différents phénomènes économiques : l'offre de marchandises rares et non reproductibles comme les tableaux, ou l'offre d'une marchandise quelconque sur une période tellement courte qu'une entreprise n'arrive pas à ajuster la quantité selon les variations du prix.

Les raisonnements de Rau ne semblent pas se référer à la firme individuelle, mais à l'industrie dans son ensemble: quoique cet aspect ne soit jamais explicitement précisé dans l'ouvrage, nous croyons pouvoir affirmer, sur la base de ce qui précède, que la droite verticale représente la quantité d'une marchandise disponible à un moment donné sur le marché; elle 
correspond donc à l'offre globale de cette marchandise. L'étude de Rau se situe alors d'emblée à un niveau agrégé, sans établir un lien précis entre le marché et l'entreprise.

\subsection{La courbe d'offre croissante}

L'interprétation de la courbe croissante $e k$ suggérée par Rau ressemble à celle de Hermann : «Il se trouve que le coût de production n'est pas toujours le même pour tous les vendeurs d'un bien. De ce fait, les cas suivants peuvent se présenter : 1) Lorsque les vendeurs supportant le coût de production le plus bas peuvent produire n'importe quelle quantité de la marchandise, ils vont être les seuls à vendre, et la concurrence entre eux fait en sorte que le prix se fixe au niveau correspondant à leur coût de production. 2) Si ces vendeurs ne peuvent offrir qu'une quantité limitée de la marchandise, le prix est déterminé par l'intensité de la demande. 2a) Si la quantité demandée est telle qu'elle peut être fournie par les producteurs qui supportent le coût le plus bas, seuls ceux-ci vont pouvoir continuer l'activité productive, et la quantité apportée sur le marché va être petite. 2b) En revanche, lorsque la demande est tellement élevée, que cette partie des producteurs s'avère insuffisante, le prix doit augmenter jusqu'au niveau correspondant au coût de production des autres vendeurs, et les entrepreneurs qui produisent à un coût moindre vont obtenir un gain » (Rau (1841), pp. 171-2). Il est très clair dans ce cas que l'analyse de Rau est centrée sur la branche et non pas sur la firme, et s'expose à la critique de Sraffa qui a été évoquée au sujet de Hermann.

La possibilité de coûts de production croissants est précisée davantage dans un passage ajouté lors d'une édition postérieure. Les coûts de production peuvent être croissants en raison de la nécessité d'avoir recours à des produits provenant de régions éloignées, ce qui ajoute aux coûts de production de base une dépense pour le transport : «Dans la plupart des cas, on peut porter sur le marché une provision plus élevée seulement à des coûts supérieurs, surtout en raison d'une distance plus grande» (Rau (1868), p. 210). En outre, les différences naturelles de sol, climat, etc. peuvent rendre la production plus coûteuse dans certaines régions : "Dans d'autres cas, un produit peut être obtenu dans un endroit donné à des conditions meilleures, ou à des coûts moindres, qu'ailleurs, de telle sorte que certains producteurs ont un avantage par rapport aux autres » (Rau (1868), p. 212).

Comme dans le cas précédent, on peut envisager une explication alternative, en tenant compte de la période temporelle considérée. Considérons le cas d'une marchandise $i$ dont l'offre est représentée, pour la «journée de marché », par la droite $e d$. Si l'on considère une durée un peu plus longue, les obstacles mentionnés plus haut, qui empêchent à très court terme tout ajustement de l'offre, deviennent moins contraignants, ainsi permettant aux producteurs de modifier la quantité en fonction du prix. Des facteurs comme le capital et le travail, dont on ne peut guère accroître la quantité au cours 
d'une journée, peuvent être augmentés si l'on accorde le temps nécessaire. La courbe $e k$ peut alors représenter l'offre de la même marchandise $i$ à court terme. Sur cette base, on pourrait aller plus loin que Rau, et considérer qu'à long terme la quantité utilisée de tous les facteurs est susceptible d'être augmentée sans engendrer des coûts unitaires plus élevés : la courbe d'offre de $i$ va être représentée par une droite horizontale. Il s'ensuit que la même courbe correspond à de diverses situations, selon la période temporelle considérée; inversement, l'offre d'une même marchandise est représentée par des courbes différentes, selon que l'on se situe dans une optique de journée de marché, court terme ou long terme.

Certaines caractéristiques du dessin de la courbe $e k$ peuvent apparaître aujourd'hui curieuses : pourquoi le prix de toute quantité plus petite que $A e$ est égal à zéro ? Pourquoi, pour tout prix inférieur à $A a$, la quantité est toujours égale à $A e$ ? On ne saurait trouver des réponses précises chez Rau. Son diagramme traduit une notion économique (la relation croissante entre prix et quantité), mais a des propriétés qui ne correspondent à aucune idée économique bien définie; réciproquement, on trouve dans le texte de Rau des concepts et des notions économiques qui ne sont pas représentés graphiquement. La figure ne correspond qu'approximativement au raisonnement économique qui s'y accompagne.

\section{$3 \quad$ Les diagrammes de Mangoldt}

Hans Karl Emil von Mangoldt (1824-1867) étudia le droit en Allemagne et en Suisse, avant de se consacrer à l'économie. Il travailla pour l'administration publique et ensuite pour une revue, mais fut obligé de démissionner à cause de ses idées politiques. Après avoir été chargé de cours à l'université de Göttingen, il fut nommé professeur à Freiburg. Nous allons nous concentrer sur son Grundriß der Volkswirthschaftslehre, un manuel de théorie économique dans lequel il utilisa des graphiques représentant l'interaction de l'offre et de la demande dans un cadre d'équilibre partiel, ainsi que quelques équations. Nous allons nous intéresser surtout à ses diagrammes, particulièrement significatifs pour notre problématique.

\subsection{L'analyse partielle}

Contrairement à Rau, Mangoldt énonça explicitement la condition «toutes choses égales par ailleurs », qui justifie l'approche d'équilibre partiel. Il supposa «pour traiter de la valeur d'échange d'un seul type de bien et de ses variations, la constance de la valeur et donc des rapports de valeur entre les objets échangés, en prenant en compte uniquement les variations de la valeur d'échange du bien, qui sont provoquées par des circonstances qui agissent directement sur celle-ci » (Mangoldt (1863), p. 46). Seuls les éléments qui 
influencent directement le marché $i$ doivent être pris en considération pour déterminer le prix et la quantité d'équilibre de la marchandise $i$, toutes les influences indirectes (c'est-à-dire, celles qui passent par des ajustements sur les marchés $j \neq i$ ) étant négligeables. Tout se passe comme si l'équilibre sur le marché considéré était réalisé indépendamment des processus conduisant à l'équilibre sur les autres marchés. Mangoldt ne s'interrogea pas davantage sur la pertinence de cette approche; il faut attendre la contribution de Marshall pour une étude plus approfondie des avantages et des limites de l'analyse partielle.

\subsection{Les coûts constants}

Mangoldt utilisa, comme Rau, des représentations sur le plan cartésien, avec les prix en ordonnées et les quantités en abscisses. Rau ne traça pas la courbe relative au cas des coûts constants, tout en reconnaissant que celuici est possible: «Lorsque les vendeurs supportant le coût de production le plus bas peuvent produire n'importe quelle quantité de la marchandise, ils vont être les seuls à vendre, et la concurrence entre eux fait en sorte que le prix se fixe au niveau correspondant à leur coût de production » (Rau (1841), p. 171). Mangoldt construisit ce diagramme (fig. 2) : «L'offre d'un bien n'est normalement possible que sous la condition que le prix de revient compense le sacrifice fait pour la réalisation de ce bien, à savoir le coût de production, c'est pourquoi le point de départ de la ligne de l'offre est le point correspondant de l'échelle des prix. [...]. La quantité offerte peut augmenter, à ce même prix, jusqu'au niveau correspondant à un changement du coût de production; jusqu'à ce point, la ligne d'offre est une droite horizontale » (Mangoldt (1863), p. 49).

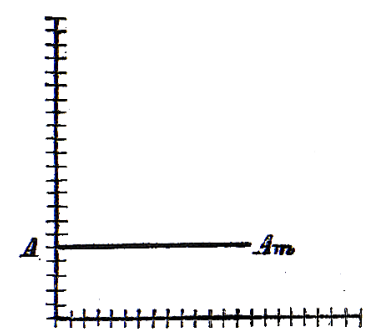

Figure 2 les coûts constants chez Mangoldt ((1863), p. 49).

Dans ce cas, le prix est déterminé par l'offre, indépendamment de la demande : «Les prix des biens, dont l'offre peut être augmentée à n'importe quel niveau, sont déterminés par les coûts de production », et « Les biens, dont l'offre peut être modifiée à n'importe quel niveau, c'est-à-dire sans changement des conditions de production, s'échangent l'un contre l'autre dans le rapport qui est déterminé par leurs coûts de production » (Mangoldt (1863), p. 52). 
Comme Rau et Hermann, Mangoldt semble raisonner plus au niveau de la branche dans son ensemble qu'au niveau de la firme individuelle, sans pour autant distinguer explicitement ces deux points de vue. Par ailleurs, une courbe d'offre horizontale est acceptable pour la branche, mais pose problème si on l'interprète comme une courbe d'offre de la firme : en concurrence parfaite, le producteur considère le prix de marché comme une donnée et, sous cette hypothèse, détermine la quantité à produire, selon le critère de la maximisation du profit; mais dans ce cas la quantité est indéterminée car, pour un prix égal au coût marginal, n'importe quelle quantité comprise entre zéro et $A_{m}$ pourrait être produite, ce qui rend la maximisation du profit un critère insuffisant pour déterminer l'équilibre.

\subsection{La rareté}

La question de la rareté, soulevée par Rau pour expliquer la courbe d'offre verticale, fut reprise par Mangoldt, selon lequel elle ne concerne pas que des biens particuliers comme les œuvres d'art, mais en dernière analyse l'ensemble des marchandises, de telle sorte que toute courbe d'offre, à partir d'un point donné $A m$ (qui varie d'une marchandise à l'autre, d'un moment dans le temps à l'autre, et d'un état de la connaissance humaine à l'autre) se transforme en une droite verticale (fig. 3) : «L'offre trouve sa limite au niveau de la quantité auquel les ressources naturelles entrant dans la production sont épuisées, en un sens absolu ou relatif à l'état de la connaissance et de la richesse humaine. À partir de ce niveau, aucune hausse du prix ne saurait faire augmenter la quantité produite, et dans la représentation graphique la ligne d'offre devient une droite verticale, qui se prolonge à l'infini. L'offre de tous les biens a une telle borne, mais celle-ci peut être tellement éloignée, qu'il n'est pas nécessaire d'en tenir compte en pratique » (Mangoldt (1863), p. 50).

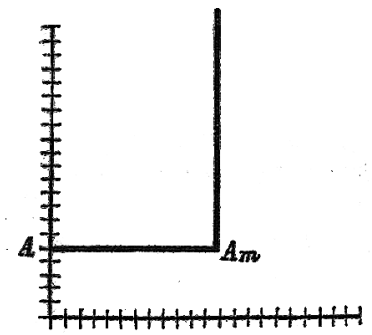

Figure 3 la rareté pour Mangoldt ((1863), p. 50).

\subsection{Les coûts variables avec la quantité produite}

Selon Mangoldt, avant d'atteindre le point au-delà duquel la quantité ne peut plus être augmentée, il peut y avoir une phase dans laquelle le coût de 
production ne reste pas constant, mais varie avec la quantité (fig. 4) : « Il se peut que la limite ne se présente pas immédiatement au niveau maximum jusqu'auquel l'offre peut être augmentée au coût de production initial, mais qu'il existe entre les deux une zone intermédiaire plus ou moins étendue, à l'intérieur de laquelle une augmentation de la production est possible à des coûts de production différents, aussi bien d'une manière telle que le coût monte, que d'une manière telle que le coût diminue avec la quantité produite, par exemple en raison des économies faites par les grandes entreprises » (Mangoldt (1863), p. 50).

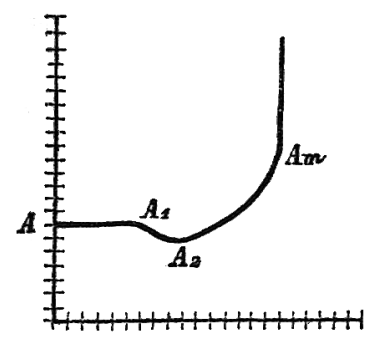

Figure 4 les coûts variables chez Mangoldt ((1863), p. 50).

La description de ce cas de figure par Mangoldt est lacunaire : elle ne donne aucune justification des coûts de production croissants, se bornant à indiquer l'une des causes des coûts décroissants, les économies réalisées par les grandes entreprises. Par ailleurs, celles-ci soulèvent un problème délicat pour un schéma concurrentiel, comme Cournot ((1838), p. 46) l'avait bien vu : lorsqu'une firme peut réduire indéfiniment son coût de production en augmentant le produit, elle finit par arriver seule à satisfaire toute la demande de marché; on se retrouve dans un cas de monopole. Ce problème n'est pas traité par Mangoldt, qui n'examina pas davantage le phénomène des coûts décroissants. En général, cette question ne semble pas avoir fait l'objet d'une analyse très approfondie à cette époque; comme Mangoldt, Rau se limita à la mentionner brièvement («pour une production plus élevée, il est possible de faire des économies » (1868), p. 210).

La courbe de la figure 4 qui se compose, entre le trait horizontal et le vertical, d'une section décroissante $A_{1} A_{2}$, suivie d'une partie croissante $A_{2} A_{m}$, peut apparaître comme une anticipation de l'idée de Marshall que la tendance à la croissance des coûts n'est dominante qu'au-delà d'un certain niveau de la production, tandis qu'en deçà, c'est la tendance aux coûts décroissants qui l'emporte. Mais il n'y a chez Mangoldt aucune explication précise de la forme en U de sa courbe. Il a peut-être voulu, tout simplement, représenter dans un seul graphique les cas des coûts croissants et décroissants, sans pour autant exclure la possibilité d'autres combinaisons (par exemple, une courbe d'abord horizontale, ensuite croissante, puis verticale, comme celle de la figure 5). 
Pour conclure, il faut souligner un mérite de Mangoldt, qui distingua clairement entre une variation du coût de production qui concerne uniquement les dernières unités produites, de telle sorte que la courbe d'offre présente une section croissante ou décroissante, et une variation des frais de production susceptible d'être étendue à l'ensemble de la production (par exemple, une baisse du coût de production en raison d'un progrès technique applicable sans limites, quel que soit le niveau d'activité), qui fait déplacer la courbe d'offre toute entière vers le haut ou vers le bas, et rapproche ou éloigne le point à partir duquel la courbe devient une droite verticale (fig. 5 : de $A S A_{m}$ à $\left.A_{1} S_{1} A_{m 1}\right)$.

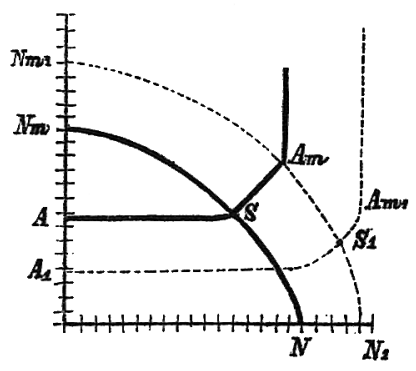

Figure 5 les déplacements de la courbe d'offre chez Mangoldt ((1863), p. 50).

\section{Conclusions}

Nous avons essayé d'expliquer la naissance et les développements pré - marginalistes de l'idée d'une relation entre prix et quantité produite. Nous avons montré que, avant Marshall, cette idée résulte de la transformation graduelle de concepts initialement formulés par les économistes classiques dans un cadre théorique différent. Le phénomène des coûts décroissants avait été traité par les classiques dans le cadre de l'étude des effets de la division du travail, et les coûts croissants dans le contexte de la théorie de la rente, sans que l'idée d'une relation fonctionnelle entre la quantité produite et le coût d'une marchandise n'émerge clairement. Les thèses classiques firent l'objet d'un travail de réinterprétation par Hermann, Rau et Mangoldt, qui essayèrent de généraliser la théorie ricardienne de la rente à quasiment toutes les branches productives, en assimilant la terre à n'importe quel type de capital fixe, et d'établir une analogie entre les entreprises qui entrent et sortent d'une branche et les terres marginales, cultivées ou non selon les circonstances. En même temps, ces auteurs concentrèrent leur attention de plus en plus sur la branche particulière plutôt que sur le système économique dans son ensemble. Cela les conduisit graduellement à mettre l'accent sur les variations du prix relatif d'une marchandise qui peuvent avoir lieu lors 
d'une augmentation de la quantité produite de celle-ci, en négligeant les effets de cette modification sur les autres variables de l'économie: la mise au point de l'idée d'une relation non proportionnelle entre la quantité produite d'un bien et son prix est associée à l'affirmation progressive de la méthode d'analyse partielle.

La construction des premières fonctions d'offre s'accompagne de l'utilisation d'outils algébriques et géométriques, repris par la suite. Les travaux examinés ici sont pourtant assez primitifs : premièrement, les potentialités des mathématiques en tant que moyens de découverte de nouvelles idées économiques ne furent pas entièrement saisies par les trois économistes allemands, qui tirèrent leurs conclusions principales de raisonnements littéraires. Deuxièmement, algèbre et géométrie restent séparées dans leurs travaux: Hermann écrivit ses formules sans tracer de graphiques, alors que Rau et Mangoldt indiquèrent les différentes formes géométriques possibles de leurs courbes sans donner les équations correspondantes. En particulier, Rau se méfiait de l'algèbre, qui avait donné à son avis des résultats insatisfaisants, et considérait la géométrie comme un outil plus puissant (« Jusqu'ici les tentatives de clarifier les effets de la concurrence sur le prix des marchandises à l'aide de formules arithmétiques n'ont pas réussi. Il est plus aisé de parvenir à cet objectif par une autre voie, la représentation géométrique » (1841), p. 581). Troisièmement, la correspondance entre contenu économique et forme mathématique est imparfaite: les graphiques de Rau et Mangoldt se limitent à indiquer que les courbes peuvent être croissantes ou décroissantes, horizontales ou verticales, sans préciser davantage leurs caractéristiques. On ne peut guère établir si Rau considérait que l'offre est toujours inélastique pour des niveaux bas du prix, ou si Mangoldt attribuait un sens précis à la forme en $U$ de la courbe de la figure 4.

Malgré ces limites, nous considérons que les mathématiques ont contribué à l'évolution de la pensée économique, en favorisant la transition de la vision classique à la néoclassique. L'interprétation économique des différents cas, qui puise dans les théories classiques, suggère la diversité et la dissymétrie: en premier lieu, la cause des coûts croissants est la présence d'un facteur rare (la terre ou tout capital fixe), tandis que la raison invoquée pour justifier les coûts décroissants (l'augmentation des dimensions de la firme) renvoie plutôt à une augmentation de la quantité produite d'un bien que l'on peut supposer en relation avec les quantités utilisées de tous les facteurs. En outre, l'analyse des coûts croissants est clairement centrée sur la branche, non sur la firme, tandis que le point de vue adopté pour l'étude des coûts constants est plus ambigu, et le passage de Mangoldt sur les coûts décroissants semble se référer davantage à l'entreprise qu'à l'industrie. En revanche, la formalisation algébrique et surtout la représentation géométrique suggèrent une vision symétrique et unitaire des différents phénomènes économiques sous-jacents à une fonction d'offre. La formule du coût de production de Hermann peut être utilisée pour exprimer aussi bien le cas des coûts constants que le cas des coûts croissants. Les graphiques tendent également vers une représentation unifiée des différents cas possi- 
bles, vus comme des courbes ayant des caractéristiques semblables, ce qui explique la possibilité qu'il existe des courbes formées par des sections différentes, comme celle de Mangoldt. La représentation géométrique « cache » pour ainsi dire l'hétérogénéité des phénomènes économiques sous-jacents, qui ne peut émerger que dans les raisonnements littéraires.

La représentation géométrico - analytique s'adapte mieux à une théorie symétrique et unitaire des différentes forces agissant sur la formation des grandeurs de marché, comme celle de Marshall, qui précisa et systématisa les idées formulées précédemment, en distinguant période de marché, courte période et longue période, et courbes d'offre de la firme et de la branche. D'ailleurs, du point de vue historique, les diagrammes d'offre et de demande sont devenus d'usage courant en économie à une époque relativement tardive par rapport à la date de leur première parution chez Rau et Mangoldt. L'invention de la représentation géométrique a chronologiquement précédé la mise au point de l'approche marshallienne, mais seul le succès de cette dernière en a véritablement permis l'affirmation et la diffusion sur une vaste échelle.

Mais la systématisation de Marshall n'a pas réussi à éviter certaines incohérences et ambiguïtés, comme le prouvent les critiques formulées par Sraffa à son égard. Quelques-unes de ces incohérences sont déjà présentes chez les précurseurs examinés ici, en raison des dissymétries de fond, héritées de la vision classique, et de la difficulté d'adapter les thèses de Smith et Ricardo à des contextes différents, dans un cadre d'équilibre partiel. Ces difficultés semblent avoir persisté dans le temps, malgré les efforts de les éliminer, et malgré une utilisation de plus en plus poussée de l'instrument géométrico - analytique. Il ne s'agissait donc pas des petites imprécisions des pionniers, éliminables grâce à un affinement progressif de l'analyse, mais de difficultés de fond, concernant le cour de la théorie, et de ce fait inéluctables, même pour les versions les plus achevées de celle-ci.

\section{Références}

BALOGLOU, C. (1995), Die Vertreter der mathematischen Nationalökonomie in Deutschland zwischen 1838 und 1871, Marburg, Metropolis.

COURNOT, A.A. (1838), Recherches sur les principes mathématiques de la théorie des richesses, réimprimé (1980), Paris, Vrin.

FRISCH, R. (1950), "Alfred Marshall's Theory of Value", Quarterly Journal of Economics, Vol. 64, nov., pp. 495-524.

GroeneweGEN, P.D., K.H. KAUfHOLD, J. SCHUMANN (1995), Hans von Mangoldt. Grundriss der Volkswirtschaftslehre. Kommentarband, Düsseldorf, Wirtschaft und Finanzen.

HENNINGS, K.H. (1980), "The Transition from Classical to Neo-Classical Economic Theory : Hans von Mangoldt", Kyklos, n. 33, pp. 658-81.

HERMANN, F.B.W. (1832), Staatswirthschaftliche Untersuchungen - über Vermögen, Wirthschaft, Productivität der Arbeiten, Kapital, Preis, Gewinn, Ein- 
kommen und Verbrauch, Munich, Anton Weber; réimprimé (1987), Düsseldorf, Wirtschaft und Finanzen.

HUMPHREY, T.M. (1992), "Marshallian Cross Diagrams and their Uses before Marshall: The Origins of Supply and Demand Geometry", Federal Reserve Bank of Richmond Economic Review, Vol. 78 (Mars/Avril), pp. 3-23.

KURZ, H.D. (1998a), "Friedrich Benedikt Wilhelm Hermann on Capital and Profits", The European Journal of the History of Economic Thought, Vol. 5, n. 1, pp. 85-119.

KURZ, H.D. (1998b), "Hermanns Beitrag zur Kapital- und Verteilungstheorie", in H.D. Kurz, Ökonomisches Denken in klassischer Tradition, Marburg, Metropolis, pp. 147-214.

KURZ, H. D. (1999), "From Classical Rent Theory to Marginal Productivity Theory. The Works of F. B. W. von Hermann and J. H. von Thünen", in G. Mongiovi et F. Petri (éds.), Value, Distribution and Capital. Essays in Honour of P. Garegnani, Londres et New York, Routledge, pp. 145-64

MANGOLDT, H. (1863), Grundriß der Volkswirthschaftslehre. Ein Leitfaden für Vorlesungen an Hochschulen und für das Privatstudium, Stuttgart, J. Engelhorn; réimprimé (1995), Düsseldorf, Wirtschaft und Finanzen.

MARshall, A. (1890), Principles of Economics, Londres, Macmillan.

PIX, M. (1999) (éd.), Friedrich Benedikt Wilhelm von Hermann (1795-1868). Ein Genie im Dienste der bayerischen Könige, Zeitschrift für bayerische Sparkassengeschichte, Beihefte, Vol. 2.

QUETELET, A. (1841), "Communication de M. Quetelet : Extraits de deux lettres, qui lui ont été adressées par M. le Professeur Rau de Heidelberg", in W.J. Baumol et S.M. Goldfeld (éds.), Precursors in Mathematical Economics : an Anthology, Londres, London School of Economics and Political Science, (1968), pp. 181-3.

RAU, K.H. (1841), Grundsätze der Volkswirthschaftslehre, 4ème edition, Heidelberg, C.F. Winter; (1ère édition 1826, 8ème edition 1868).

RECKTENWALD, H.C. (1987), Friedrich von Hermann - ein Wegbereiter moderner Theorie, Düsseldorf, Wirtschaft und Finanzen.

SCHEFOLD, B. (1997), "Einleitung", in K.H. Rau, Grundsätze der Volkswirthschaftslehre, réimpression de la première édition (1826). Hildesheim, Zürich et New York, Olms-Weidmann, pp. I - LIII.

SRAFFA, P. (1925), "Sulle relazioni fra costo e quantità prodotta", Annali di economia, n. II, pp. 277-328.

STREISSLER, E.W. (1990), "The Influence of German Economics on the Work of Menger and Marshall", in B.J. Caldwell (éd.), Carl Menger and His Legacy in Economics, Durham et Londres, Duke University Press, pp. 31-68.

STREISSLER, E.W. (1997), Friedrich Benedikt Wilhelm von Hermann : Anatomie einer wissenschaftlichen Verwandlung, Munich, Verlag der bayerischen Akademie der Wissenschaften.

THEOCHARIS, R. D. (1993), The Development of Mathematical Economics. The Years of Transition : From Cournot to Jevons, Londres, Macmillan.

VINER, J. (1931), Cost Curves and Supply Curves, Zeitschrift für Nationalökonomie, Vol. III, sept., pp. 23-46. 